\title{
Microvasculature Of The Mammalian Urinary Bladder - A Preliminary Comparative Study Using Vascular Corrosion Casting
}

\author{
F. E. Hossler*, R. L. Kao**, and F. C. Monson*** \\ Departments of Anatomy and Cell Biology* and Surgery**, J.H. Quillen College of Medicine, \\ East Tennessee State University, Johnson City, TN 37614 \\ LEXSPIAC, Large Scientific Instrument Core ***, West Chester University, West Chester, PA \\ 19320
}

The urinary bladder is a unique organ in that it is subject to considerable distension and contraction during normal function. It is well known that prolonged distension due to outflow blockage from disease compromises blood flow, and may lead to ischemia. However, because normal distension and contraction do not lead to pathology, it is of interest to know if there are specializations of the bladder vasculature that help promote blood flow to its wall during distension [1]. This study utilized routine vascular corrosion casting [2] to compare the vasculatures of the bladder in guinea pig, rabbit, pig, and dog. In each case, following anticoagulation and euthanasia with i.p. drugs, the abdominal aorta was cannulated, the vena cava was opened for blood drainage, and the lower abdominal vasculature was flushed of blood with warm $0.9 \% \mathrm{NaCl}$ at physiological pressure. The vasculature was then perfused with freshly prepared Mercox resin ( $8 \mathrm{ml}$ Mercox, $2 \mathrm{ml}$ methylmethacrylate monomer, and $0.3 \mathrm{ml}$ catalyst) until the onset of polymerization (about 10 $\mathrm{min})$. The bladder and its surrounding tissue was immersed in water $\left(60^{\circ} \mathrm{C}\right)$ overnight, the tissue was removed with alternating rinses of $5 \% \mathrm{KOH}$ and distilled water, and the casts were lyophilized. Casts were dissected apart, and portions of the casts were mounted with carbon paint, coated with gold, and viewed by routine scanning electron microscopy.

Examples of vascular casts of the bladder mucosae of the guinea pig, rabbit, pig and dog, respectively, viewed apically and from the serosal side are shown at similar magnifications in Figs. 1-4. Note that the mucosal vasculatures consist of a dense, freely anastomosing, capillary bed, that is supplied from beneath by a dense bed of highly coiled arterioles and venules. Histological sections reveal that the capillary beds lie juxtaposed to the urothelium. Other features common to most, but perhaps not all, of the bladder vasculatures studied here include: (1) extensive coiling of the supply vessels, especially at the bladder apices; (2) abundant venous valves; (3) extensive anastomoses (collateral vasculature) in the arterial and venous portions of vasculature; (4) distinct separation of the mucosal and submucosal vascular beds permitting independent movements of each during distension; (5) a perpendicular arrangement of connecting vessels between the mucosal and submucosal vascular beds to reducing stress on the vasculature during distension; (6) abundant arterial sphincters; and (7) a specialized elastic sheath enclosing some of the supplying arteries. Examples of these features will be presented, and their possible significance with regard to bladder blood flow will be discussed.

\section{References}

[1] F.E. Hossler and F.C. Monson, Anat. Rec. 243 (1995) 438.

[2] S. H. Aharinejad and A. Lametschwandtner. Microvascular Corrosion Casting, SpringerVerlag, Wien, 1992. 


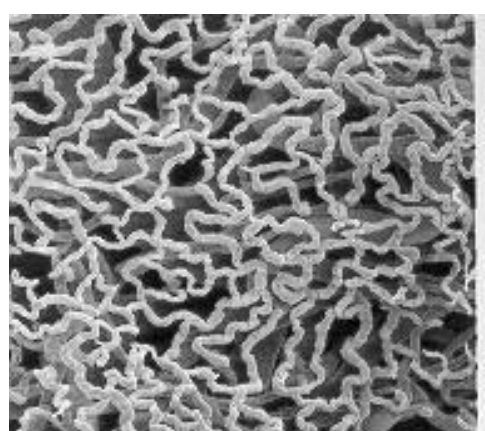

$1 \mathrm{~A}$

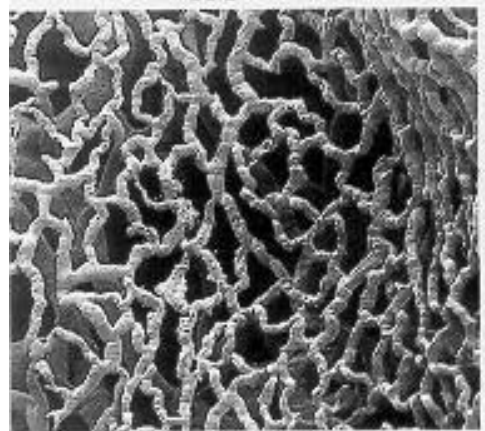
$2 \mathrm{~A}$

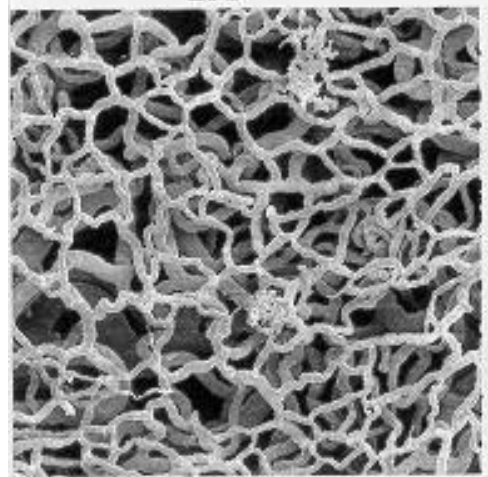

$3 \mathrm{~A}$

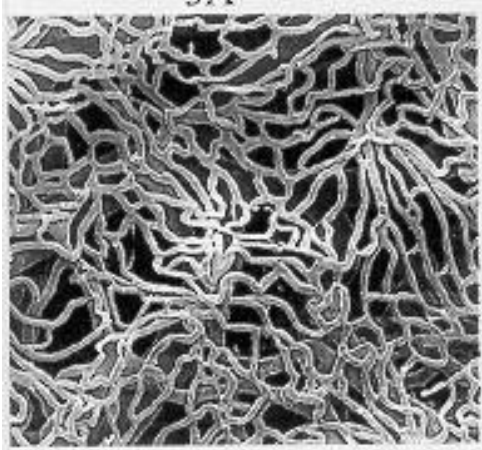

$4 \mathrm{~A}$

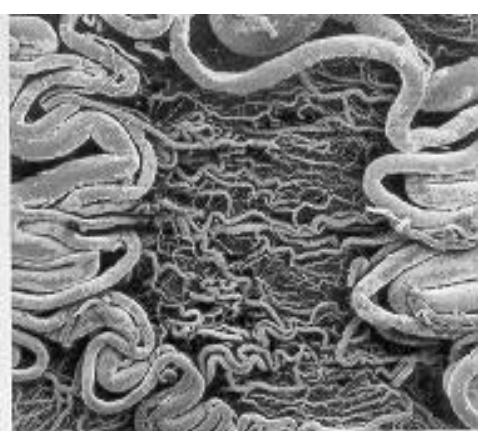

1B

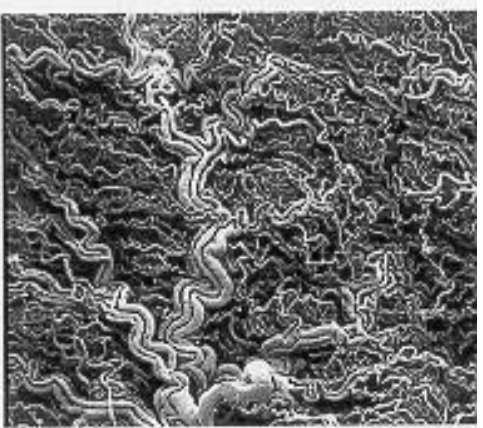

$2 \mathrm{~B}$

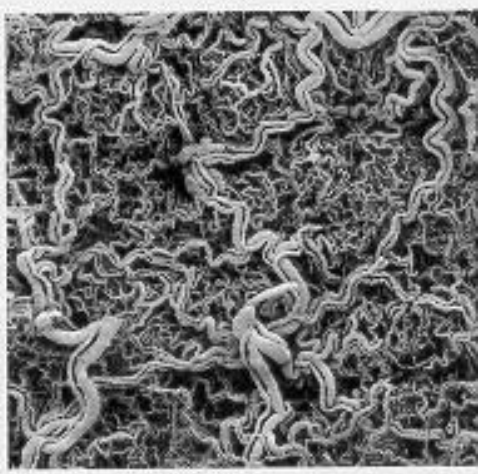

3B

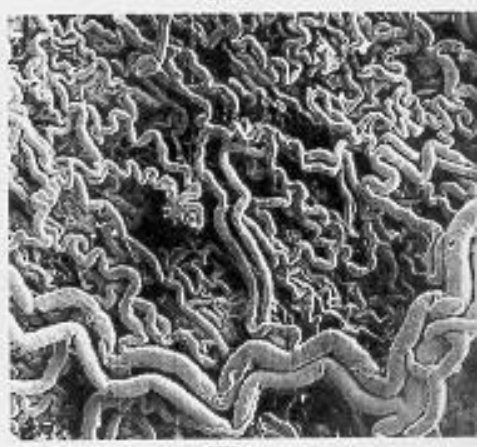

4B

Fig. 1. Guinea pig bladder mucosal vasculature: A, surface view (x100); B, serosal view (x25)

Fig. 2. Rabbit bladder mucosal vasculature: A, surface view (x200); B, serosal view (x14).

Fig. 3. Pig bladder mucosal vasculature: A, surface view (x100); B, serosal view (x20).

Fig. 4. Dog bladder mucosal vasculature: A. surface view (x75); $\mathrm{B}$, serosal view (x25). 